\title{
Chronic Pain: What Does It Mean? A Review on the Use of the Term Chronic Pain in Clinical Practice
}

This article was published in the following Dove Press journal: Journal of Pain Research

\author{
William Raffaeli $\mathbb{B D}^{\prime}$ \\ Michael Tenti ${ }^{1}$ \\ Annette Corraro ${ }^{2}$ \\ Valentina Malafoglia' \\ Sara Ilari ${ }^{3}$ \\ Eleonora Balzani (iD ${ }^{4}$ \\ Antonello Bonci ${ }^{1,5}$
}

'ISAL Foundation, Institute for Research on Pain, Rimini, Italy; ${ }^{2}$ Leiden University, Faculty of Social and Behavioural Sciences, Leiden, The Netherlands; ${ }^{3}$ Institute of Research for Food Safety \& Health (IRC_FSH), Department of Health Sciences, University 'Magna Graecia' of Catanzaro, Catanzaro, Italy; ${ }^{4}$ Department of Surgical Science, University of Turin, Torino, Italy; ${ }^{5} \mathrm{Global}$ Institutes on Addictions, Miami, FL, USA
Correspondence: William Raffaeli

Fondazione ISAL, Via San Salvador, 204,

Rimini, 47922, Italy

Tel +390541725166

$\mathrm{Fax}+390541725164$

Email isal@fondazioneisal.it

\begin{abstract}
Chronic pain is nowadays used as an umbrella term referring to a wide range of clinical conditions, such as fibromyalgia, migraine, or long-standing pain states without actual known causes. However, labeling a patient's clinical condition with the term "chronic pain", when dealing with pain lasting longer than 3 months, might be misleading. This paper aims at analyzing the possible pitfalls related to the use of the term "chronic pain" in the clinical field. It appears, indeed, that the term "chronic pain" shows a semantic inaccuracy on the basis of emerging scientific evidences on the pathogenesis of different long-standing pain states. The major pitfalls in using this label emerge in clinical settings, especially with patients having a biomedical perspective on pain or from different cultures, or with healthcare providers of other medical specialties or different disciplines. A label solely emphasizing temporal features does not help to discern the multifaceted complexity of long-standing pain states, whose onset, maintenance and exacerbation are influenced by a complex and interdependent set of bio-psycho-social factors. Thus, finding a more meaningful name might be important. We call upon the necessity of bringing awareness and implementing educational activities for healthcare providers, as well as for the public, on the biopsychosocial approach to assess, prevent and care of chronic pain. Further research on the etiopathogenetic processes of chronic pain states is also required, together with examinative diagnostic methods, to individuate the most appropriate label(s) representing the complex longstanding pain states and to avoid adopting the term "chronic pain" inappropriately.
\end{abstract}

Keywords: chronic pain, disease, diagnosis, label, meaning, biopsychosocial

\section{Introduction}

The International Association for the Study of Pain (IASP) defines chronic pain as persistent or recurrent pain lasting longer than 3 months. ${ }^{1}$ It represents a major healthcare problem worldwide, affecting $19 \%$ of European adults ${ }^{2}$ and $20.4 \%$ of US adults. ${ }^{3}$ Chronic pain is not a mere temporal extension of acute pain, as it lacks the warning function of physiological nociception, and it is maintained by factors pathogenetically and physically remote from the initial cause, such as central sensitization, altered pain modulation, glial activation, and neuroimmune signaling. ${ }^{4-6}$ The development and chronicization of pain can be also influenced by several psychological and social factors, such as depression, catastrophizing, avoidance behaviors, somatization, responses from significant others and cultural attitudes. ${ }^{7}$ The reciprocal interaction of biological and psychosocial elements suggests the multifaceted nature of chronic pain, and the urgency of a biopsychosocial 
approach for its assessment, prevention and management. ${ }^{8}$ In addition to being maintained by an interdependent set of bio-psycho-social factors, chronic pain is often accompanied by several biological (eg, depression of the immune system), physical (eg, impaired functioning), psychological (eg, depression) and social consequences (eg, job loss), which all contribute in aggravating the patient's burden. ${ }^{9}$ The stated evidences, together with the great social and economic load of chronic pain on healthcare systems and societies, ${ }^{10-13}$ led several stakeholders worldwide in asking governments to place chronic pain as a public health priority. $^{12,14}$ More recently, chronic pain disorders have been also categorized systematically into the 11th version of the International Classification of Diseases (ICD-11), with the intention of strengthening the representation of these dramatic conditions in clinical practice and research. $^{15}$

Chronic pain is nowadays used as an umbrella term for referring to a wide range of painful conditions, such as fibromyalgia, migraine, or long-standing pain states having an unclear etiology. In the field of research, there are several reasons to use "chronic pain" as umbrella term across diagnoses, especially in epidemiological studies. For example, major comorbidities, outcomes and psychosocial burden frequently depend more on the severity and interference of pain than on the diagnosis itself. ${ }^{16}$ Nevertheless, in clinical settings, the term "chronic pain" may be problematic if used as label for the patient's clinical condition, in particular for pain lasting more than 3 months and lacking clear explanations. The term "chronic pain" may appear troublesome especially for patients sharing a mind-body dualism or from different cultures, since it exclusively emphasizes the time criterion to explain the morbid nature of the problem. This results in an incomplete explanation of the reasons how a condition, usually known as a symptom of a damaged body part, persists without a clear underlying biological cause. As previously mentioned, chronic pain is the outcome of a dynamic interaction among biological, psychological and social factors, which perpetuates and aggravates the pain itself, individual disability and the response to interventions. ${ }^{8}$ In the clinical encounter, the use of term "chronic pain" may lead to difficulties in understanding the multifaceted complexity of long-standing pain states, and even producing frictions between the physician and the patient, assuming his/her clinical condition to be misinterpreted. Patients may indeed find the label "chronic pain" as being complicated to grasp, as part of them might consider impossible to have daily pain without any underlying disease. ${ }^{17}$ Clarke and Iphofen ${ }^{18}$ suggested that for several patients the lack of an acceptable label for their long-standing pain becomes the "cloak of invisibility" that hides suffering from the outside world. "Chronic pain" is a label which might produce misunderstandings also among doctors of different medical specialties (eg, rheumatology, neurology, orthopedics) and healthcare providers of different disciplines (eg, physiotherapists, psychologists, chiropractors), since each of them perceives pain based on the knowledge and skills they acquired during their academic education.

Therefore, the aim of this paper is to analyze the use of the term "chronic pain" in clinical settings when dealing with pain lasting longer than 3 months without actual known causes, in order to highlight the possible pitfalls. In the first section of this paper, we present a historical reconstruction of the evolution of the term "chronic pain" and of its uses and meanings. Placing our debate within a broader historical context can help to better understand how the meanings associated to this term have evolved over time and what meanings it has today. Then, we provide some reflections on the term "chronic pain" and its meanings, especially considering the pivotal role played by the adjective "chronic". Finally, we raise the question on whether a more meaningful term should be adopted and suggest tips and initiatives which could help us to enhance patients' understanding of their own painful condition and to legitimize their experience.

\section{Chronic Pain: An Historical Reconstruction of Its Uses and Meanings}

As noted by Baszanger, ${ }^{19}$ before the 1950 s pain persisting for long time with obscure etiology or when the primary cause has already been identified and appropriately managed, constituted a residual category of medical practice. By performing a literature research on Google Scholar using the keyword "chronic pain" in the title, and with time limit 1900-1950, only 28 results are retrieved, and none of them refers to chronic pain as a specific clinical condition without any primary cause to treat. The ItaloAmerican anesthesiologist John J. Bonica was the first who called attention to this type of pain in its textbook "The Management of Pain". ${ }^{20}$ Here, the father of pain medicine started to draw attention on the specificity of the so-called "intractable pain", which, in his view, had 
to be considered as a pathological entity with a destructive force given the serious consequences on patients' health and quality of life. Another term often used by Bonica was "abnormal", to indicate a type of pain exceeding the normal one of brief duration. In our opinion, Bonica probably used the terms "intractable" and "abnormal" as at that time the lack of biological function of that pain and the complete failure of therapies was evident and frustrating. The term "chronic" was used rarely by Bonica, and mainly as a synonymous of "persistent" or to indicate a poor prognosis.

It is noteworthy that in Bonica's view the intractable and abnormal pain represented a pathological entity because of its devastating physical and mental consequences on patients that relied mainly on its persistence. The extended duration of pain was therefore considered as a crucial aspect of the problem. ${ }^{20}$

As Baszanger ${ }^{19}$ suggested, the term "chronic pain" began to be popular during the first international symposium on pain organized by Bonica in Issaquah in 1973. In fact, in the second part of the conference, six papers mentioned "chronic pain" in their titles. ${ }^{21-26}$ However, none of them referred to chronic pain as a peculiar medical condition or disease: one paper used "chronic pain" referring to pain behaviors, ${ }^{22}$ while the others used that term as synonymous of "persistent", "recurrent", "unremitting", "intractable" or "long-standing".

The year following the Issaquah conference, Richard A. Sternbach dedicated an entire chapter to chronic pain in his book "Pain Patients: traits and treatment". ${ }^{27}$ In his work, Sternbach described chronic pain as a diverse experience from that of the acute pain, mostly due to its dramatic psychological consequences. However, unlike other authors, ${ }^{19}$ we believe that Sternbach used the term "chronic pain" to group different diseases characterized by pain of long duration with a great burden for the individual, and not to indicate a peculiar type of pain. In fact, he clearly stated that examples of chronic pain states may be causalgia, thalamic pain, trigeminal neuralgia, etc. and that "patients with these diseases usually experience constant pain". 27

A few years later, Pilowsky stated that "whereas in acute pain the pain is the symptom of a disease, in chronic pain the pain is itself the disease". ${ }^{28}$ Thus, the author used the term "chronic pain" to indicate a peculiar pathological state, albeit not entirely explicitly. However, the complex issue of chronic pain as a symptom or disease began to emerge.
In the second edition of "The Management of Pain", for the first time, "chronic pain" became a clear label indicating pain

Which persists a month beyond the usual course of an acute disease or reasonable time for an injury to heal, or pain that recurs at intervals for months or years. ${ }^{29}$

At the Second Congress of the Italian Society of Pain Clinicians held in 1992, Raffaeli used the term "chronic pain" for referring explicitly to an autonomous pathology, namely a "chronic pain status" characterized by the sole and imperative presence of pain requiring a therapeutic response. ${ }^{30}$ In the late nineties, given the advances in the scientific discoveries related to neurobiological mechanisms underlying many long-standing pain states, Michael Cousins proposed "chronic pain" as the disease of the 21 st century, ${ }^{31}$ while the EFIC defined "chronic pain" as a disease in its own right. ${ }^{9}$ In the meantime, Siddall and Cousins $^{32}$ used the label "persistent pain" (by referring to pain that persists beyond 3 months) to describe a type of pain which represents a disease entity in itself, characterized by its own pathology, symptoms and signs (eg, peripheral and central sensitization, secondary changes in primary afferent nerves, alteration of the autonomic nervous system). From this moment on, the term "chronic pain" was increasingly used also to indicate a disease in its own right.

Also, Tracey and Bushnell ${ }^{5}$ present a review of works showing compelling evidence of functional, structural, and chemical changes in the brain in association with chronic pain, thus finally supporting the idea that chronic pain should be placed "in the realm of a disease state". Likewise, several authors suggested to view "chronic pain" as a brain disease, despite this conception is rather controversial and debated. ${ }^{33}$

Starting from the third edition of "The Management of Pain", the reference to the temporal criterion, as well as to the strict link between chronic pain and the initial cause, were eliminated from the definition of chronic pain. ${ }^{34}$ However, the temporal criterion remains in the IASP definition, that is "persistent or recurrent pain lasting longer than 3 months".

\section{Chronic Pain: Some Reflections on the Term and Its Meanings}

Based on the above historical reconstruction, it appears that the term "chronic pain" started to be adopted in the 
seventies, while pain medicine was also founded, probably becoming crucial in delineating the problem encountered by clinicians at that time: a very intractable and persistent pain. This term was initially used referring to pain regarded as a symptom or to group different diseases with a similar prevailing clinical manifestation, ie, pain which persists over a long period of time, even after the resolution of the generating cause. However, over the years, "chronic pain" has also been increasingly used to define a disease in its own right. Consequently, it is clear that a term identifying both a symptom and a disease risks to create ambiguity and confusion.

Besides, the term "chronic pain" could be semantically inaccurate, as label for patient's clinical condition, when dealing with pain lasting longer than 3 months without actual known causes.

In our opinion, the critical issues in using this term to define this type of pain are related to the adjective "chronic". The word "chronic" derives from the Greek "khronikos" and refers merely to the passage of time itself. $^{35}$ The Oxford English Dictionary defines the term "chronic" relating to diseases as "lasting a long time" and "opposed to acute". However, long-standing pain conditions are not just something opposed to those of acute pain, as they are also accompanied by characteristic neurobiological changes such as central sensitization, altered pain modulation, glial activation and neuroimmune signaling, ${ }^{4-6}$ and are much more influenced by biopsycho-social factors. ${ }^{7,8}$ Moreover, from a biological perspective, the long duration is not the defining aspect of complex long-standing pains. For example, patients who experienced a stroke involving the spinothalamic system may develop contralateral hemibody pain which may persist for the rest of their life. As suggested by some authors, ${ }^{36,37}$ the pathological character of this kind of pain does not develop over time, but it is "chronic" from its onset. Even according to the IASP, the time criterion is not a substantial characterization of chronic pain syndromes and a certain flexibility in the comparison of cases is suggested. ${ }^{38}$ The temporal reference of 3/6 months was also aimed at prompt physicians to pursue further investigation to exclude the presence of etiopathological factors not detected initially, which would anyway manifest in a time lapse coherent to that of other pathologies. However, 3/6 months are sometimes insufficient to evaluate all the possible underlying causes of pain, and the time needed to identify its possible causes may also depend on the organizational variables of the healthcare system taken into account. Referring to the time criterion may not be entirely accurate for another reason, namely that the mere fact of not having found any causes does not mean that there are no causes, but that they may not have been discovered yet. To date, it is not possible to know which of the two hypotheses is the most accurate. ${ }^{39}$

By considering "chronic" in the sense of an adjective, it only describes a quality of pain, thus not promoting its own specificity as it continues to refer to the universally known concept of pain, namely the physiological response to danger for the organism. The meaning of the adjective "chronic" is clearer when used next to terms referring to pathological processes of a specific organ's function such as "nephritis". The Chronic Obstructive Pulmonary Disease (COPD) is a further example. Here, the adjective "chronic" is not misleading as the other terms already refer to a clear pathological process (in this case, obstructive) affecting the lungs. The term "pain" does not describe any organ or system, nor a pathological process in itself, rather it is typically recognized as a symptom of another injury or disease. Hence, by merely adding the term "chronic", only one quality of this symptom is described, that is the irreversibility and the long duration over time. A study ${ }^{40}$ found that many patients with no coronary artery disease who received the diagnosis of "non-cardiac chest pain" remain doubtful about the diagnosis and limited in their activity. Authors suggest that for these patients it could be easier to deal with a known, acceptable illness, ie, a heart disease, rather than the vaguer diagnosis of chronic chest pain of unknown origin.

The term "chronic pain", especially when used as label to indicate a pain condition viewed as a disease in itself, may also carry the risk of a delay in the diagnosis of the underlying pathology and inappropriate treatment. Tamburin et $\mathrm{al}^{41}$ reported a case of a middle-aged woman with well-controlled diabetes mellitus and a severe chronic pain (NRS ranging from 8 to 10/10) in the left lower quadrant of the abdomen. After an accurate neurological investigation, the initial diagnosis of chronic abdominal pain has been replaced with that of neuropathic pain, due to thoracic radiculopathy and probable lumbosacral radiculoplexus neuropathy, and the following corticosteroid therapy resulted in consistent pain reduction (NRS reduced to $2-3 / 10$ ).

The use of the term "chronic pain" might result insidious in clinical settings, especially with patients dealing with pain lasting more than 3 months without known causes. Raffaeli et $\mathrm{al}^{17}$ noted that patients with chronic 
non-cancer pain, compared to those with cancer pain, often fail to make sense of their pain, because they may have difficulties to understand that it is possible to have daily pain without any other disease causing it. A qualitative study, involving young people with chronic pain and their parents, found that "chronic pain" - as diagnostic label may elicit uncertainty and was sometimes perceived as a diagnosis given when the biological cause of pain has not been identified, or when pain is not deemed "real", thus producing frictions within the patient-clinician relationship. $^{42}$ In the same study, lacking evidence of physical causes, patients and their parents struggled to make sense of a diagnosis of "chronic pain". Moreover, several participants expressed concerns that a serious health condition (eg, cancer) could have been missed, which fuelled a relentless search for alternative diagnosis. In the field of pain, an unclear diagnostic label is found to be associated with negative clinical, psychological and social outcomes as well as with further uncertainty and treatment seeking, which may place an extra burden on health services and prevent patients from directing their attention to other aspects of life. ${ }^{4,44}$ Therefore, "chronic pain", as diagnostic label, could not facilitate the comprehension of the clinical condition. Furthermore, despite the patient recognizes long-standing pain as a more complex health problem, the term "chronic pain" might continue to reinforce a dualistic vision in family members, who, having likely experienced pain as a transient condition, may also stigmatize certain pain behaviors as a source of "social disruption": the sick role is legitimate only for a period of time. ${ }^{45}$

As suggested by the anthropologist Jean Jackson, ${ }^{45}$ chronic pain does not fit into the shared notion about the nature of pain, viewed as a transient symptom of an underlying injury or disease. In our opinion, this perspective is sustained by the rooted belief of pain as a symptom coming from a precise underlying biological source, present in patients, laypeople, and health professionals, despite the biopsychosocial model demonstrated to be the most heuristic approach to chronic pain assessment, prevention, and treatment. 8,39

Other pitfalls associated with the term "chronic pain", when used as label for patient's clinical condition, can be identified from a cultural perspective. The origin of chronic diseases finds a different explanation as we encounter different cultures: while in the Western world it is often explained as caused by biological factors, other cultures give a metaphysical explanation. Consequently, some cultures might believe that chronic pain is caused by god's will, witchcraft, karma, family's sins and aging, thus considering pain not as a medical problem to treat but as a payment for a certain behavior or as a natural occurring. ${ }^{46}$ Other cultures are linked with the concept of hardship and resilience; thus, they refrain western biomedical interventions to relief pain. Besides, another element is pain as a sign of weakness and shame, and cultures believing it do not generally seek for aid. ${ }^{47}$ Consequently, the term "chronic pain", as a label, may have different effects on patients from different cultures, for example in their understanding of the problem or adherence to treatments. In order to deal with a multicultural society, such diversifications should be carefully considered in choosing the most appropriate terminology.

\section{The Importance to Give a Name (A Meaningful One)}

The above reflections suggest that the term "chronic pain" hides several pitfalls if used for labeling patient's clinical condition when dealing with pain lasting longer than 3 months without actual known causes. The same reflections also raise the question on whether a more meaningful term should be adopted.

An attempted redefinition of chronic pain was carried out by the American Academy of Pain Medicine (AAPM) a few years ago, ${ }^{48}$ when proposing the definition of "maldynia" referring to a neurologic condition characterized by pathologic changes in the nervous system and resembling that of chronic pain. The term "maldynia" has been used since it allows patients to better grasp the impact of neurobiological changes, to highlight a need for action and to minimize pejorative and judgmental viewpoints regarding their experience of lasting pain. Therefore, it seems that the AAPM acknowledged the poor explanatory capacity of "chronic pain" as a label for complex long-standing pain without actual known causes. However, this conceptualization has been criticized since the distinction between "good" and "bad" pain (ie, maldynia vs eudynia) risks to be moralizing and stigmatizing for both patients and clinicians. $^{49}$ Other authors ${ }^{50-52}$ have recently introduced the label "pathological pain" for referring to pain lasting longer than 3 months clinically and characterized by spontaneous pain, hyperalgesia and allodynia, or "mysterious central pains" for referring to pain states (eg, fibromyalgia, noncardiac chest pain) that are not associated with 
a neurological deficit but still presumed to be due to central nervous system dysfunction. ${ }^{37} \mathrm{Nijs}$ et $\mathrm{al}^{53}$ raised also the question on the usefulness of the term "central sensitization" as a label for a subgroup of patients with severe and spreading pain. In our opinion, they might represent interesting attempts of redefinition, drive by the poor explanatory capacity and pitfalls of the term "chronic pain" for lasting pain without actual known causes.

Several chronic pain conditions represent a disease state that needs a specific name. The adjective "chronic" merely describes a feature of the disease and it should not be used as a name for a disease. As an example, the diabetes is a chronic disease, but its name is "diabetes", not "chronic diabetes".

It is difficult to find a name that effectively represents lasting pain without actual known causes since they have not yet been fully clarified its etiological mechanisms. The problem also lies in the inadequacy of our current technology to identify the sources of persistent pain. However, in our opinion, the term "chronic pain" may result problematic beyond this technological limit, since it requires an accurate explanation of its meaning, within a biopsychosocial approach. This is not a problem in itself, because providing information about the disease and its predisposing, precipitating, perpetuating and protective factors is a fundamental aspect of the patient-provider communication. Chronic pain represents a "biopsychosocial experience", but, as described above, the term "chronic pain" identifies both a symptom and a disease, and the belief of pain as a symptom due to a precise underlying biological source is deeply rooted in patients and public opinion, and in many health professionals as well. ${ }^{39}$ Therefore, a biopsychosocial explanation for chronic pain can easily be misunderstood in cultural contexts where the mind and the body represent separate and non-communicating entities, and may lead patients to believe that their pain, for doctors, is only in their mind, or that the doctor did not understand the clinical condition. The short time of the clinical encounter as well as the lack of provider continuity in public settings may easily result in similar misunderstandings, which risk being reinforced by family members and even healthcare providers, especially when they argue the need for a multidisciplinary approach in theory, and later preferring an essentially biomedical (or psychogenic) approach in practice.

An alternative term to "chronic pain" does not avoid the necessity of an explanation, which is fundamental regardless the terms used, but could reduce equivocacy.
The ICD-11 introduced the label "chronic primary pain" to avoid the obsolete dichotomy of "physical" vs "psychological" ${ }^{54}$ However, this label just adds another adjective to the term pain, highlighting another quality (that of not being linked to another cause), thus not eliminating the possible ambiguity associated with the term "pain".

The term "chronic pain" is more immediate than the others, as it could be, for example, "Bonica's disease", a label chosen just to pay homage to the father of pain medicine. While we move this provocation, we strongly believe this label to be less equivocal, therefore reducing the possibility of misunderstandings.

Probably, it could be better to use the more generic label "chronic pain syndrome", as originally suggested by IASP, until their exact underlying causes are better understood. In this way, some authors have recently suggested to group chronic pain disorders into the new ICD-11 under a category of its own called "Disorders/Diseases Related to Chronic Pain" ${ }^{55}$ or returning to the peripherally driven or centrally maintained dichotomy already proposed by Bonica in the fifties. ${ }^{37}$

As noted by Gallagher, ${ }^{56}$ medical terminologies are crucial to effectively connect a disease to the minds and hearts of the public and policy makers. Nowadays, several individuals still believe that a person with "hypertension" is a very tense person. It is therefore necessary to find a name as clear as possible that leaves no room for interpretation. Probably, what hinders the recognition of a more representative label (or labels) is an ultimate etiological description of the etiopathogenetic processes underlying the different chronic pain states. Despite we still do not know all about pain, in 2018 the IASP formed a multinational task force to evaluate its current definition and accompanying note, and whether they should be retained or changed. ${ }^{57}$ In our opinion, given the advances in our understanding of complex long-standing pain states, the development of a task force to evaluate the appropriateness of the label "chronic pain" could be taken into consideration. The debate on the appropriateness of this label might also involve patients' opinions. As the sufferers experience the symptoms of the disease, they might be the most representative in individuating the label that reflects their condition, and that allows them to match their identity with. A study has been conducted on Chronic Fatigue patients having to determine the name that could better substitute the label "Medical Unexplained Symptoms", a term that refers to a variety of diseases 
often presenting distinct symptomatology. In the study, participants not only preferred specific terms depending on how they appropriately evoked the disease and the symptoms, but also on their cultural background influences. ${ }^{58}$ Nonetheless, the point of view of the patients has not been enough investigated yet, thus this study should open to further research, in particular for what concerns "chronic pain". Besides, paying attention to the cultural background is not only essential as it impacts the decision-making during the research, but also because as the world is becoming more and more multicultural, finding a solution that tries to satisfy different perspectives is fundamental to increase the functionality of the healthcare system.

\section{Conclusion}

In the present work, we have retraced the evolution of the term "chronic pain", initially chosen to merely define pain of long duration and difficult to treat, or to group different diseases with a similar prevailing clinical manifestation. Today, it is often used as a label for patient's clinical condition when the underlying causes of pain have not been identified, and pain is viewed as the sole medical problem to treat. However, "chronic pain" hides several pitfalls in clinical settings, if used as a diagnostic label for this type of pain. Time is not the crucial aspect of pain lasting longer than 3 months, as it is associated with several biological alterations, ${ }^{4-6}$ as well as having numerous psychological and social elements contributing to its onset, maintenance, and exacerbation. ${ }^{7,8}$ The term "chronic pain" may show pitfalls especially in clinical settings, as several patients, who might share a biomedical perspective and a mind-body dualism, do not consider pain to exist without an underlying disease, and consequentially, the vagueness of the term might favor distress and further treatment seeking. ${ }^{43}$ Moreover, the term "chronic pain" may trigger different comprehensions and reactions in patients from different cultures. ${ }^{46,47}$

The term "chronic pain" adopted to define complex long-standing pain without actual known causes had surely a great political significance in the process of legitimation of pain medicine. However, as pain research progresses, it is likely to be misleading, especially for patients.

We call clinicians (physicians, psychologists, nurses, etc.) upon the necessity of avoiding ambiguous and imprecise terminologies as much as possible. The use of a vague terminology may lead to difficulties in grasping the multifaceted complexity of long-standing pain states, and even producing frictions between the physician and the patient, assuming his/her clinical condition to be misinterpreted. Therefore, if a clear name for the patient's clinical condition exists, then that name should be adopted. Otherwise, if a generic term such as "chronic pain" is adopted, then exploring the meanings that the patient attributes to her/his condition is crucial. Labelling patient's pain generically as "chronic pain" may fail to incorporate the range of factors that can contribute to the condition, limiting its understanding and affecting the management strategies that the patient may decide to adopt. As suggested in the literature, we encourage clinicians to create time for providing patient comprehensible information about chronic pain and on the biopsychosocial approach to its management, in order to foster a positive therapeutic alliance and positive health outcomes. ${ }^{59}$ It is noteworthy that patients are able to appreciate reconceptualizations of chronic pain from a more integrated perspective, although health professionals may underestimate their ability to understand. ${ }^{60}$

In parallel, we believe social awareness initiatives are needed to enhance public understanding of long-standing pain states, their causes and management strategies, within a biopsychosocial approach. As recommended by the Institute of Medicine, education of health care providers is another crucial part of the necessary cultural transformation of the approach to chronic pain. ${ }^{12}$ Particularly, we claim the need for implementing pain curriculum for health care providers and to increase the number of health professionals with advanced expertise in chronic pain care, within a biopsychosocial approach to its assessment, prevention and treatment. Finally, more research is needed on etiological basis of chronic pain conditions as well as on diagnostic methods for neurobiological mechanisms underlying them, from wet biomarkers ${ }^{61}$ to other techniques. ${ }^{62}$ This is the only way to find the more meaningful label - or labels - and, equally important, to avoid applying the "chronic pain" diagnosis as the outcome of mere process of exclusion. While waiting for progresses of research, as suggested by Gallagher,

It remains to be seen how the language of pain will evolve with the science of pain medicine to help bring bench to the bedside in this long-underfunded field of clinical research. ${ }^{56}$

\section{Disclosure}

The authors report no conflicts of interest in this work. 


\section{References}

1. Treede RD, Rief W, Barke A, et al. A classification of chronic pain for ICD-11. Pain. 2015;156(6):1003-1007. doi:10.1097/j. pain.0000000000000160

2. Reid KJ, Harker J, Bala MM, et al. Epidemiology of chronic non-cancer pain in Europe: narrative review of prevalence, pain treatments and pain impact. Curr Med Res Opin. 2011;27 (2):449-462. doi:10.1185/03007995.2010.545813

3. Dahlhamer J, Lucas J, Zelaya C, et al. Prevalence of chronic pain and high-impact chronic pain among adults - United States, 2016. MMWR Morb Mortal Wkly Rep. 2018;67(36):1001-1006. doi:10.15585/ mmwr.mm6736a2

4. Watkins LR, Milligan ED, Maier SF. Glial activation: a driving force for pathological pain. Trends Neurosci. 2001;24(8):450-455. doi:10.1016/s0166-2236(00)01854-3

5. Tracey I, Bushnell MC. How neuroimaging studies have challenged us to rethink: is chronic pain a disease? J Pain. 2009;10 (11):1113-1120. doi:10.1016/j.jpain.2009.09.001

6. Grace PM, Hutchinson MR, Maier SF, Watkins LR. Pathological pain and the neuroimmune interface. Nat Rev Immunol. 2014;14 (4):217-231. doi:10.1038/nri3621

7. Turk DC, Gatchel RJ, Eds. Psychological Approaches to Pain Management: A Practitioner's Handbook. 3rd ed. New York: The Guilford Press; 2018.

8. Bevers K, Watts L, Kishino ND, Gatchel RJ. The biopsychosocial model of the assessment, prevention, and treatment of chronic pain. US Neurol. 2016;12(2):98-104. doi:10.17925/USN.2016.12.02.98

9. Niv D, Devor M. Chronic pain as a disease in its own right. Pain Pract. 2004;4(3):179-181. doi:10.1111/j.1533-2500.2004.04301.x

10. Lipman AG. Pain as a human right: the 2004 global day against pain. J Pain Palliat Care Pharmacother. 2005;19(3):85-100.

11. Saastamoinen P, Laaksonen M, Kääriä SM, et al. Pain and disability retirement: a prospective cohort study. Pain. 2012;153(3):526-531. doi:10.1016/j.pain.2011.11.005

12. Simon LS. Relieving pain in America: a blueprint for transforming prevention, care, education, and research. Mil Med. 2016;181 (5):397-399. doi:10.7205/MILMED-D-16-00012

13. Breivik H, Eisenberg E, O'Brien T; OPENMinds. The individual and societal burden of chronic pain in Europe: the case for strategic prioritisation and action to improve knowledge and availability of appropriate care. BMC Public Health. 2013;13:1229. doi:10.1186/ 1471-2458-13-1229

14. Hanna M. Pain in Europe: a public health priority. $J$ Pain Palliat Care Pharmacother. 2012;26(2):182-184. doi:10.3109/15360288 .2012 .681839

15. Treede RD, Rief W, Barke A, et al. Chronic pain as a symptom or a disease: the IASP classification of chronic pain for the international classification of diseases (ICD-11). Pain. 2019;160(1):19-27. doi:10.1097/j.pain.0000000000001384

16. Steingrímsdóttir ÓA, Landmark T, Macfarlane GJ, Nielsen CS. Defining chronic pain in epidemiological studies: a systematic review and meta-analysis. Pain. 2017;158(11):2092-2107. doi:10.1097/j. pain.0000000000001009

17. Raffaeli W, Andruccioli J, Florindi S, et al. Qualitative pain classification in hospice and pain therapy unit. Am J Hosp Palliat Care. 2012;29(8):604-609. doi:10.1177/1049909111435810

18. Clarke KA, Iphofen R. A phenomenological hermeneutic study into unseen chronic pain. Br J Nurs. 2008;17(10):658-663. doi:10.12968/ bjon.2008.17.10.29482

19. Baszanger I. Inventing Pain Medicine: From the Laboratory to the Clinic. New Brunswick, New Jersey, and London: Rutgers University Press; 1988.

20. Bonica JJ. The Management of Pain. Philadelphia: Lea \& Febiger; 1953.
21. Fordyce WE. Pain viewed as learned behavior. Adv Neurol. 1974;4:415-422.

22. Greenhoot JH, Sternbach RA. Conjoint treatment of chronic pain. Adv Neurol. 1974;4:595-603.

23. Halpern LM. Psychotropic drugs and the management of chronic pain. Adv Neurol. 1974;4:539-545.

24. Houde RW. The use and misuse of narcotics in the treatment of chronic pain. Adv Neurol. 1974;4:527-536.

25. Long DM, Carolan MT. Cutaneous afferent stimulation in the treatment of chronic pain. Adv Neurol. 1974;4:755-759.

26. Sato T, Nakatani Y. Acupuncture for chronic pain in Japan. $A d v$ Neurol. 1974;4:813-818.

27. Sternbach RA. Pain Patients: Traits and Treatments. New York: Academic Press; 1974.

28. Pilowsky I. Chronic pain as a disease entity. Triangle. 1981;20 (1-2):27-32.

29. Bonica JJ. The Management of Pain. Philadelphia: Lea \& Febiger; 1990.

30. Raffaeli W. Il dolore. L'antalgologia: storia di una scienza medica in attesa di una propria identità specialistica. Atti del Secondo Congresso Nazionale SICD Bressanone. [The Antalgologia: history of a medical science waiting for its specialistic identity. Acts of the Second National Congress of the Italian Society of Pain Clinicians]. In: Rizzi R, Raffaeli W, Pari G, Butera G, editors. Atti Congresso Nazionale SICD. Bressanone;1992.

31. Cousins MJ. Pain: the past, present, and future of anesthesiology? The E. A. Rovenstine memorial lecture. Anesthesiology. 1999;91 (2):538-551. doi:10.1097/00000542-199908000-00029

32. Siddall PJ, Cousins MJ. Persistent pain as a disease entity: implications for clinical management. Anesth Analg. 2004;99(2). doi:10.1213/01.ANE.0000133383.17666.3A

33. Sullivan MD, Cahana A, Derbyshire S, Loeser JD. What does it mean to call chronic pain a brain disease? J Pain. 2013;14(4):317-322. doi:10.1016/j.jpain.2012.02.012

34. Loeser JD, Chapman CR, Turk DC, Butler SH, eds. Bonica's Management of Pain. Lippincott Williams \& Wilkins; 2000.

35. Dowrick C, Dixon-Woods M, Holman H, Weinman J. What is chronic illness? Chronic Illn. 2005;1(1):1-6. doi:10.1177/ 17423953050010010901

36. Raffaeli W, Arnaudo E. Pain as a disease: an overview. J Pain Res. 2017;10:2003-2008. doi:10.2147/JPR.S138864

37. Loeser JD. A new way of thinking about pain. Pain Manag. 2019;9 (1):5-7. doi:10.2217/pmt-2018-0061

38. Merskey H, Bogduk N. Classification of Chronic Pain, IASP Task Force on Taxonomy. Seattle, WA: International Association for the Study of Pain Press; 1994.

39. Raffaeli W. Du symptôme douleur à la maladie douleur. Chronique d'une transformation incertaine. Rev Sci Soc. 2015;53:10-16.

40. Ockene IS, Shay MJ, Alpert JS, Weiner BH, Dalen JE. Unexplained chest pain in patients with normal coronary arteriograms: a follow-up study of functional status. N Engl J Med. 1980;303(22):1249-1252. doi:10.1056/NEJM198011273032201

41. Tamburin S, Magrinelli F, Zanette G. Diagnostic and therapeutic pitfalls in considering chronic pain as a disease. Pain Med. 2014;15 (9):1640-1642. doi:10.1111/pme.12495

42. Neville A, Jordan A, Beveridge JK, Pincus T, Noel M. Diagnostic uncertainty in youth with chronic pain and their parents. $J$ Pain. 2019;20(9):1080-1090. doi:10.1016/j.jpain.2019.03.004

43. Serbic D, Pincus T. Chasing the ghosts: the impact of diagnostic labelling on self-management and pain-related guilt in chronic low back pain patients. J Pain Manag. 2013;6(1):25-35.

44. Serbic D, Pincus T. Diagnostic uncertainty and recall bias in chronic low back pain. Pain. 2014;155(8):1540-1546. doi:10.1016/j.pain.2014.04.030

45. Jackson JE. Stigma, liminality, and chronic pain: mind-body borderlands. Am Ethnol. 2005;32(3):332-353. doi:10.1525/ae.2005.32.3.332

46. Groce NE, Zola IK. Multiculturalism, chronic illness, and disability. Pediatrics. 1993;91(5 Pt 2):1048-1055. 
47. Pillay T, van Zyl HA, Blackbeard D. Chronic pain perception and cultural experience. Procedia Soc Behav Sci. 2014;113:151-160. doi:10.1016/j.sbspro.2014.01.022

48. Dickinson BD, Head CA, Gitlow S, Osbahr AJ 3rd. Maldynia: pathophysiology and management of neuropathic and maladaptive pain-a report of the AMA council on science and public health. Pain Med. 2010;11(11):1635-1653. doi:10.1111/j.1526-4637.20 10.00986.x

49. Cohen M, Quintner J, Buchanan D. Is chronic pain a disease? Pain Med. 2013;14(9):1284-1288. doi:10.1111/pme.12025

50. Kuner R. Central mechanisms of pathological pain. Nat Med. 2010;16(11):1258-1266. doi:10.1038/nm.2231

51. Luo C, Kuner T, Kuner R. Synaptic plasticity in pathological pain. Trends Neurosci. 2014;37(6):343-355. doi:10.1016/j.tins.2014.0 4.002

52. Sun J, Chen F, Braun C, et al. Role of curcumin in the management of pathological pain. Phytomedicine. 2018;48:129-140. doi:10.1016/ j.phymed.2018.04.045

53. Nijs J, Polli A, Willaert W, Malfliet A, Huysmans E, Coppieters I. Central sensitisation: another label or useful diagnosis? Drug Ther Bull. 2019;57(4):60-63. doi:10.1136/dtb.2018.000035

54. Nicholas M, Vlaeyen JWS, Rief W, et al. The IASP classification of chronic pain for ICD-11: chronic primary pain. Pain. 2019;160 (1):28-37. doi:10.1097/j.pain.0000000000001390

55. Lu Y, Cheng J, Han JS, et al. A proposal to add a new dedicated chapter in ICD-11: disorders related to chronic pain. Pain Med. 2020;21(3):436-438. doi:10.1093/pm/pnz060

56. Gallagher RM. Chronification to maldynia: biopsychosocial failure of pain homeostasis. Pain Med. 2011;12(7):993-995. doi:10.1111/ j.1526-4637.2011.01186.x
57. Raja SN, Carr DB, Cohen M, et al. The revised international association for the study of pain definition of pain: concepts, challenges, and compromises. Pain. 2020;161(9):1976-1982. doi:10.1097/j.pain.0 000000000001939

58. Picariello F, Ali S, Moss-Morris R, Chalder T. The most popular terms for medically unexplained symptoms: the views of CFS patients. J Psychosom Res. 2015;78(5):420-426. doi:10.1016/j. jpsychores.2015.02.013

59. Dorflinger L, Kerns RD, Auerbach SM. Providers' roles in enhancing patients' adherence to pain self management. Transl Behav Med. 2013;3(1):39-46. doi:10.1007/s13142-012-0158-Z

60. Moseley L. Unraveling the barriers to reconceptualization of the problem in chronic pain: the actual and perceived ability of patients and health professionals to understand the neurophysiology. J Pain. 2003;4(4):184-189. doi:10.1016/s1526-5900(03)00488-7

61. Raffaeli W, Malafoglia V, Bonci A, et al. Identification of MOR-positive $\mathrm{B}$ cell as possible innovative biomarker $(\mathrm{Mu}$ Lympho-Marker) for chronic pain diagnosis in patients with fibromyalgia and osteoarthritis diseases. Int J Mol Sci. 2020;21(4):1499. doi:10.3390/ijms21041499

62. Hackshaw KV, Aykas DP, Sigurdson GT, et al. Metabolic fingerprinting for diagnosis of fibromyalgia and other rheumatologic disorders [published correction appears in J Biol Chem. $2020 \mathrm{Apr}$ 24;295(17):5834]. J Biol Chem. 2019;294(7):2555-2568. doi:10.1074/jbc.RA118.005816
Journal of Pain Research

\section{Publish your work in this journal}

The Journal of Pain Research is an international, peer reviewed, open access, online journal that welcomes laboratory and clinical findings in the fields of pain research and the prevention and management of pain. Original research, reviews, symposium reports, hypothesis formation and commentaries are all considered for publication. The manuscript management system is completely online and includes a very quick and fair peer-review system, which is all easy to use. Visit http:// www.dovepress.com/testimonials.php to read real quotes from published authors. 